\title{
Neurodegeneration, Neuronal Loss, and Neurotransmitter Changes in the Adult Guinea Pig with Perinatal Asphyxia
}

\author{
GUENTHER BERNERT, HARALD HOEGER, WILHELM MOSGOELLER, \\ DORIS STOLZLECHNER, AND BARBARA LUBEC \\ Departments of Pediatrics [G.B., D.S.] and Neonatology [B.L.] and Institute for Cancer Research [W.M.], \\ University of Vienna, A-1090 Vienna, Austria; and Institute for Animal Breeding, University of Vienna, \\ A-2235 Himberg, Austria [H.H.]
}

\begin{tabular}{|c|c|}
\hline \multicolumn{2}{|c|}{ ABSTRACT } \\
\hline $\begin{array}{l}\text { There is only limited morphologic information on long-term } \\
\text { alterations and neurotransmitter changes after perinatal asphyxia, } \\
\text { and no long-term study showing neurodegeneration has been } \\
\text { reported so far. We used an animal model for perinatal asphyxia } \\
\text { well documented in the rat to investigate the guinea pig as a } \\
\text { species highly mature at birth. Cesarean section was performed } \\
\text { on full-term pregnant guinea pigs, and pups, still in membranes, } \\
\text { were placed into a water bath at } 37^{\circ} \mathrm{C} \text { for asphyxia periods from } \\
2 \text { to } 4 \text { min. Thereafter pups were given to surrogate mothers and } \\
\text { examined at } 3 \text { mo of age. We studied brain areas reported to be } \\
\text { hypoxia-sensitive. Neurodegeneration was evaluated by fluoro- } \\
\text { jade, neuronal loss by Nissl, reactive gliosis by glial fibrillary } \\
\text { acidic protein staining, and differentiation by neuroendocrine- } \\
\text { specific protein C immunoreactivity. We tested tyrosine hydrox- } \\
\text { ylase, the vesicular monoamine transporter, and dopamine } \beta \text {-hy- } \\
\text { droxylase, representing the monoaminergic system; the vesicular } \\
\text { acetylcholine transporter; and the excitatory amino acid carrier } 1 \text {. } \\
\text { Neurodegeneration was evident in cerebellum, hippocampal area } \\
\text { CA1, and hypothalamus, and neuronal loss could be observed in } \\
\text { cerebellum and hypothalamus; gliosis was observed in cerebel- } \\
\text { lum, hippocampus, hypothalamus, and parietal cortex; dediffer- }\end{array}$ & $\begin{array}{l}\text { entiation was found in hypothalamus and striatum; and mono- } \\
\text { aminergic, cholinergic, and amino acidergic deficits were shown } \\
\text { in several brain regions. The major finding of the present study } \\
\text { was that neurodegeneration and dedifferentiation evolved in the } \\
\text { guinea pig, a species highly mature at birth. The relevance of this } \\
\text { contribution is that a simple animal model of perinatal asphyxia } \\
\text { resembling the clinical situation of intrauterine hypoxia-ischemia } \\
\text { and presenting with neurodegeneration was characterized. } \\
\text { (Pediatr Res 54: 523-528, 2003) }\end{array}$ \\
\hline
\end{tabular}

PA continues to be a major cause of neurologic injury in the newborn, leading to reversible and irreversible brain damage ranging from minimal brain dysfunction to death (1-3). After a hypoxic-ischemic insult certain neuron subpopulations are known to die whereas others survive (4-6). This phenomenon, referred to as selective vulnerability, occurs in adult and neonatal brain. Neurons in the hippocampus, cerebellum, striatum, thalamus, and neocortex have been identified to be vulnerable to hypoxic-ischemic insults.

Received January 29, 2001; accepted September 19, 2002.

Correspondence: Barbara Lubec, M.D., Department of Neonatology, University of Vienna, Waehringer Guertel 18, A-1090 Vienna, Austria; e-mail: gert.lubec@akh-wien.ac.at

Financial support by the Red Bull Company, Salzburg, Austria.

DOI: 10.1203/01.PDR.0000081760.48331.7A
Apart from acute cell death the question arises whether PA can provoke detrimental effects on the CNS later in life, e.g. as induction of neurodegeneration. There are no long-term studies available reporting on neurodegeneration after PA, and therefore we included a marker for the detection of degenerating cells. Because only subtle morphologic differences between normal and degenerating cells may exist and thus degenerating neurons could be missed easily, we performed FJ staining as the staining pattern of this dye corresponds to the pattern of argyrophilia, staining cell bodies, dendrites, and axon terminals of degenerating neurons but not healthy neurons, myelin, vascular elements, or neuropil (7).

In addition we examined neurotransmitter-specific cell markers involved in the pathologic mechanisms of PA and candidates as mediators for long-term sequelae. We tested 
vMAT, DBH, TH, EAAC1, a neuronal subtype of the glutamate transporter, and $\mathrm{vAChT}$ using immunohistochemistry on brain sections of guinea pigs with graded PA. PA-related changes of the monoaminergic system have been reported by several groups, and the sensitivity of this system to asphyctic damage was emphasized in man and in animal models $(8-10)$, mainly revealing short- and long-term dopaminergic and $\mathrm{TH}$ deficits (11-14). With the exception of recent studies on the effect of nicotine on the pathophysiology of PA, indicating an interaction between the cholinergic and the monoaminergic neurons (10), the only systematic study on cholinergic neurons after PA was reported by Burke et al. (15): in a quantitative morphologic analysis the authors described a $23 \%$ decrease of cholinergic neurons at the age of $4 \mathrm{wk}$ and a long-lasting cholinergic deficit in the striatum. Information on the role of excitatory amino acids in PA is limited, and only few groups have been stressing the problem of glutamatergic functions (16-24).

The aim of the present study was to characterize neurodegeneration, gliosis, neuronal loss, and differentiation, as well as alterations within functional systems, as characterized by specific neurotransmitter immunoreactivity in a model of PA in an animal mature at birth.

\section{METHODS}

\section{Experimental Design}

PA was induced in pups delivered by cesarean section from pregnant guinea pigs (D.H. Him, Dunkin-Hartley, Institute for Animal Breeding, Himberg, Austria; primiparous, 105-115 g body weight). As duration of pregnancy considerably varies in guinea pigs - dependent on the number of pups - the day of cesarean section was fixed after palpation of the symphysis, which develops a gap more than $1 \mathrm{~cm}$ a few hours before parturition by relaxation of the pubic ligaments. The day of cesarean section was fixed by the same experimenter in all animals to avoid subjective bias. Animals were then killed by decapitation and hysterectomized. The uterine horns, still containing the fetuses, were extirpated, and pups used as controls were taken out of their membranes, dried, and kept in a hood; pups for the induction of PA were placed in a water bath at $37^{\circ} \mathrm{C}$ for various periods of 2, 3, 4, or $5 \mathrm{~min}$. Exposure to $5 \mathrm{~min}$ of asphyxia was not compatible with survival. After the asphyctic period the uterine horns were rapidly opened and the pups removed. Pups were cleaned, the umbilical cord was ligated, and the animals were mildly resuscitated and stimulated and allowed to recover in an incubator at $37^{\circ} \mathrm{C}$. Then pups were given to foster mothers and were well accepted. Only litters with pups with a body weight of more than $60 \mathrm{~g}$ at the time of delivery were used in the experiments $(25,26)$. Foster mothers and pups were kept in Makrolon type IV cages $\left(1800 \mathrm{~cm}^{2}\right)$ and weaned at the age of $14 \mathrm{~d}$. Animal studies were performed according to the rules of the American Physiology Society and following local ethical law. For all examinations performed at 3 mo of age, 10 animals per group (normoxic and 2,3 , and 4 min of asphyxia) were used. The experimenter was blinded in the experiments and tests.
In parallel experiments brain lactate and brain $\mathrm{pH}$ were evaluated in six animals of each group $10 \mathrm{~min}$ after delivery to allow resuscitation and thus survival and at 3 mo after the induction of PA using methods published previously $(27,28)$. Although animals showed gradual increase of brain lactate and decrease of pH shortly after delivery (Table 1), animals at 3 mo did not show any differences among groups (data not shown).

\section{Histologic Examinations}

Animals used for histologic studies at the age of 3 mo were anesthetized (Membumal, $50 \mathrm{mg} / \mathrm{kg}$ body weight, i.p.) and

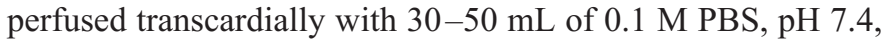
containing 4\% paraformaldehyde. Approximately 50-60 min after perfusion the brain was removed from the skull, postfixed in the same solution for $12-18 \mathrm{~h}$, and then kept in PBS containing $20 \%$ sucrose at $4^{\circ} \mathrm{C}$, pending sectioning. Paraffin embedding, preparation of $20-\mu \mathrm{m}$ sections, and dewaxing by xylene are described elsewhere (29).

FJ staining. We used the method described by Schmued and Bowyer (7) for paraffin-embedded material. Paraffin-embedded brains were sectioned at $10 \mu \mathrm{m}$, mounted on glass slides, dewaxed, and rehydrated through graded alcohols. A 15-min pretreatment with $0.06 \%$ potassium permanganate solution was followed by a brief rinse in distilled water, a 60 -min incubation in $0.001 \% \mathrm{FJ}$ in $0.1 \%$ acetic acid, and three 1 -min rinses in distilled water. The sections were then air-dried on a slide warmer, cleared in xylene, and coverslipped with $p$-xylenebis( $N$-pyridinium bromide) nonfluorescent plastic mounting media. Potassium permanganate was obtained from Aldrich Chemical Co. (Kindler Ltd, Freiburg, Germany), FJ from Histo-Chem (Jefferson, AR, U.S.A.), and $p$-xylene-bis( $N$ pyridinium bromide) from Fluka (Buchs, Switzerland). The tissue was analyzed and photographed using a Leica epifluorescence microscope with a FITC filter cube (blue light excitation). Degeneration of neuronal perikarya and fibers was recognized by their brighter fluorescence. Typically the FJ signal appeared homogeneous over the perikarya indicating a loss of subcellular internal microstructure. The number of stained cells in a given area was evaluated.

Nissl staining. Sections were dewaxed in three changes of xylene, followed by three changes of $96 \%$ ethanol for $5 \mathrm{~min}$ each. Then slides were incubated in $0.1 \%$ Luxol fast blue overnight at $60^{\circ} \mathrm{C}$, rinsed briefly in $96 \%$ ethanol, and differentiated in $0.05 \%$ lithium carbonate. For the staining of cell nuclei and cytoplasmic ribosomal RNA (Nissl substance), we used cresyl fast violet $(0.1 \% \mathrm{wt} / \mathrm{vol}, \mathrm{pH}$ adjusted to 3.9 with acetic acid; Fluka) for $3 \mathrm{~min}$, followed by two changes of

Table 1. Brain $\mathrm{pH}$ and brain lactate levels $10 \mathrm{~min}$ after period of asphyxia

\begin{tabular}{lcc}
\hline & Brain $\mathrm{pH}$ & Brain lactate levels \\
\hline Controls & $7.1 \pm 0.2$ & $6.05 \pm 1.7 \mathrm{mM} / \mathrm{g}$ brain \\
PA of $2 \mathrm{~min}$ & $7.0 \pm 0.1$ & $11.22 \pm 3.5 \mathrm{mM} / \mathrm{g}$ brain* \\
PA of $3 \mathrm{~min}$ & $6.8 \pm 0.2^{*}$ & $16.56 \pm 3.3 \mathrm{mM} / \mathrm{g}$ brain* \\
PA of $4 \mathrm{~min}$ & $6.5 \pm 0.2^{*}$ & $19.22 \pm 4.0 \mathrm{mM} / \mathrm{g}$ brain* \\
\hline
\end{tabular}

$n=6$ per group.

* Significantly different from control value using Mann-Whitney $U$ or Kruskal-Wallis test. 
distilled water and two rinses in $96 \%$ ethanol and differentiation in dried ethanol for $6 \mathrm{~min}$. After incubation in xylene, slides were mounted with a coverslip in Eukitt.

\section{Immunohistochemical Examinations}

Paraffin sections mounted on silanized glass slides were dewaxed in three changes of xylene for $15 \mathrm{~min}$ each, rehydrated by decreasing concentrations of ethanol, and briefly rinsed in distilled water and in PBS, pH 7.4, twice. For antigen retrieval the sections were incubated in $2 \mathrm{mM}$ EDTA in PBS, $\mathrm{pH} 8.0$, at $95^{\circ} \mathrm{C}$ for $60 \mathrm{~min}$, strictly avoiding any bubbling as a result of cooking. Then the slides were allowed to cool down, washed in PBS, and preincubated with blocking solution $(5 \%$ BSA, $0,3 \%$ Tween-20, and $0.3 \%$ Triton-X in PBS). The sections were incubated with the first antibody at $40^{\circ} \mathrm{C}$ for $16 \mathrm{~h}$. We used immunoglobulins diluted in blocking solution against GFAP (Dako, Glostrup, Denmark), diluted 1:500; vMAT (Chemicon, Temecula, CA, U.S.A.), diluted 1:600; TH (Chemicon), diluted 1:400; EAAC1 (Chemicon), diluted 1:500; vAChT (Chemicon), diluted 1:1000; and DBH (Affinity Research, Mamhead, U.K.), diluted 1:200. After three washings in PBS the following incubations to detect immunoglobulins were performed with an automated immunostainer (Labvision Autostainer, Fremont, CA, U.S.A.) using speciesspecific biotinylated secondary antibodies.

The color reaction consisted of incubation with 3,3-diaminobenzidine (DAB) and hydrogen peroxidase to obtain a brown precipitate. Diluted hematoxylin was applied for $2 \mathrm{~min}$ to obtain a light counterstaining of cell nuclei, followed by dehydration in ethanol series and coverslipping with Eukitt. Photomicrographs were taken on a Nikon Optiphot using a Nikon Coolpix digital camera (Nikon Instruments Europe B.V., Badhoevedorp, The Netherlands). Antibodies against vACHT, DBH, and EAAC1 preferably stained neuronal perikarya whereas vMAT and TH predominantly stained fibers.

\section{Morphometry}

Sample selection and morphometric analysis basically followed the principle of Burck (30), and quantification of the immunohistochemical signal was carried out with a computer system (Lucia V.3.52a; Laboratory Imaging, Prague, Czech Republic) measuring optical density as either number of stained cells or stained area per field of interest. Brains investigated in this study were histologically screened before analysis. Regions known to be hypoxia-sensitive in man and rat $(23,24,29,31-33)$ have been selected.

Brain regions were identified following the nomenclature of Roessner (34). Areas for the micrograph of each specific region were selected at low magnification to avoid biased selection (randomized within the brain region). Then micrographs were taken at higher magnification to cover a representative field size of the area and to enable positive identification of structures within the field. Depending on the specific staining density of cells, fibers or perikarya were determined for each investigated area in all brains. Density of degenerating neurons stained by FJ was determined in a randomly selected field of
$80,000 \mu \mathrm{m}^{2}$ in all regions except striatum, in which we used a field size of $40,000 \mu \mathrm{m}^{2}$ as overall cell density was increased. Neuronal density based on Nissl staining was determined along neuronal layers in fields of the following size: cerebellum, Purkinje cell density along $290 \mu \mathrm{m}$; CA1 pyramidal layer along $570 \mu \mathrm{m}$; hypothalamic nucleus ventromedialis within $160,000 \mu \mathrm{m}^{2}$; striatum (globus pallidus) and parietal cortex within $46,500 \mu \mathrm{m}^{2}$.

GFAP-expressing astrocytes were determined in a field of $9,640 \mu \mathrm{m}^{2}$ for cerebellum, $22,400 \mu \mathrm{m}^{2}$ for CA1 and hypothalamus, $17,800 \mu \mathrm{m}^{2}$ for striatum, and $19,700 \mu \mathrm{m}^{2}$ for the pyramidal layer of the parietal cortex. Immunoreactivity of vMAT staining was determined for Purkinje cells in L8 of cerebellum along $600 \mu \mathrm{m}$, for fibers in the hypothalamic ventromedial nucleus and globus pallidum of striatum in a field size of $67,500 \mu \mathrm{m}^{2}$, and for immunoreactive cells in the pyramidal layer of the parietal cortex in a field size of 67,500 $\mu \mathrm{m}^{2}$. Immunoreactive DBH-stained cells were determined according to Purkinje cells along a bar of $800 \mu \mathrm{m}$, neurons in the pyramidal layer CA1 were counted along a stretch of $540 \mu \mathrm{m}$, neurons in the ventromedial hypothalamic nucleus within a field of $194,000 \mu \mathrm{m}^{2}$, and globus pallidus in striatum and pyramidal layer (striatum, parietal cortex) within a field of $70,700 \mu \mathrm{m}^{2}$. TH immunoreactivity was determined as fibers per field counting $52,700 \mu \mathrm{m}^{2}$ per brain region as shown in Table 1. EAAC1 and vAChT antibody stained perikarya in fields of $39,000 \mu \mathrm{m}^{2}$ in the same regions as counted for DBH. NSP-C antibodies stained preferentially perikarya of Purkinje cells, which were analyzed under the same conditions as DBH.

\section{Statistical Calculations}

Means and SD were calculated. For the comparison of groups, morphometric data were calculated using ANOVA with a subsequent Kruskal-Wallis test.

\section{RESULTS}

FJ staining. FJ-stained cells representing degenerating neurons were significantly increased in the cerebellum in the 3and 4-min asphyctic group, and in the CA1 region of the hippocampus and in the nucleus ventromedialis of the hypothalamus in all asphyctic groups. In the parietal cortex FJ staining was increased at 2 and $3 \mathrm{~min}$. Results are given in Table 2, and an example is shown in Figure 1.

Nissl staining. Neuronal loss could be observed in the cerebellum in the 3- and 4-min asphyctic groups, in the hypothalamic nucleus ventromedialis in all asphyctic groups, and in the striatum in the 2-and 3-min asphyctic groups. Results are given in Table 2, and a representative sample is shown in Figure 1.

GFAP staining. A significant increase of GFAP staining could be found in the cerebellum and CA1 in all asphyctic groups, in the hypothalamus and cortex in the 3-min asphyctic animals, and in the striatum in the 2-min asphyctic group only. Results are given in Table 2.

$\boldsymbol{N S P}$ - C. NSP-C-immunoreactive cells were significantly decreased in the nucleus ventromedialis of the hypothalamus and 
Table 2. Histologic and immunohistologic examination of different brain regions ${ }^{\dagger}$

\begin{tabular}{|c|c|c|c|c|c|c|c|c|c|c|}
\hline $\begin{array}{c}\text { Region/ } \\
\text { minutes of } \\
\text { apshyxia }\end{array}$ & $\mathrm{N}$ & FJ & Nissl & GFAP & NSP-C & vMAT & $\mathrm{DBH}$ & $\mathrm{TH}$ & EAAC1 & vAChT \\
\hline \multicolumn{11}{|l|}{$\mathrm{Cb}$} \\
\hline $\mathrm{Co}$ & 10 & $1 \pm 0.9$ & $9.09 \pm 2.2$ & $48.79 \pm 12.4$ & $34.27 \pm 6.9$ & $19.82 \pm 4.5$ & $35.8 \pm 6.4$ & $16.8 \pm 5.2$ & $19.4 \pm 3.5$ & $33.33 \pm 5.2$ \\
\hline $2 \min$ & 11 & $1.73 \pm 1.0$ & $8.69 \pm 2.9$ & $66.71 \pm 14.2^{*}$ & $36.36 \pm 8.0$ & $14.8 \pm 5.6^{*}$ & $34.8 \pm 5.1$ & $12.18 \pm 4.9 *$ & $16.0 \pm 2.7^{*}$ & $32.3 \pm 6.3$ \\
\hline $3 \mathrm{~min}$ & 12 & $3 \pm 1.3^{*}$ & $6.58 \pm 2.9 *$ & $69.27 \pm 16.5^{*}$ & $37.0 \pm 6.9$ & $14.75 \pm 5.1^{*}$ & $32.1 \pm 4.0$ & $8.82 \pm 3.0^{*}$ & $17.58 \pm 3.4$ & $24.58 \pm 6.0^{*}$ \\
\hline $4 \mathrm{~min}$ & 15 & $3.91 \pm 1.8^{*}$ & $4.93 \pm 2.1 *$ & $90.08 \pm 19.1^{*}$ & $34.18 \pm 6.9$ & $12.83 \pm 5.9^{*}$ & $25.4 \pm 3.3^{*}$ & $11.1 \pm 4.3^{*}$ & $13.36 \pm 3.1 *$ & $22.11 \pm 4.6^{*}$ \\
\hline \multicolumn{11}{|l|}{ CA1 } \\
\hline $\mathrm{Co}$ & 10 & $5.00 \pm 3.3$ & $36.45 \pm 10.8$ & $29.23 \pm 6.8$ & $11.0 \pm 5.1$ & No signal & $70.8 \pm 10.8$ & $13.25 \pm 7.5$ & $17.2 \pm 5.3$ & $17.44 \pm 14.2$ \\
\hline $2 \min$ & 11 & $16.18 \pm 8.6^{*}$ & $39.13 \pm 9.2$ & $49.47 \pm 9.3^{*}$ & $9.73 \pm 3.5$ & & $66.4 \pm 15.5$ & $16.69 \pm 5.7$ & $17.73 \pm 3.3$ & $58.6 \pm 7.5^{*}$ \\
\hline $3 \mathrm{~min}$ & 12 & $15.91 \pm 4.7 *$ & $38.08 \pm 11.6$ & $54.72 \pm 7.9^{*}$ & $10.2 \pm 4.1$ & & $65.4 \pm 14.4$ & $16.2 \pm 7.8$ & $17.08 \pm 4.1$ & $58.41 \pm 10.6^{*}$ \\
\hline $4 \mathrm{~min}$ & 15 & $11.4 \pm 5.3^{*}$ & $40.33 \pm 12.5$ & $56.84 \pm 14.1^{*}$ & $8.8 \pm 4.1$ & & $55.5 \pm 12.2^{*}$ & $13.34 \pm 8.7$ & $11.7 \pm 4.6^{*}$ & $55.38 \pm 15.9^{*}$ \\
\hline \multicolumn{11}{|l|}{ Ht } \\
\hline $\mathrm{Co}$ & 10 & $0.36 \pm 0.7$ & $8.36 \pm 3.5$ & $29.92 \pm 6.1$ & $7.09 \pm 2.5$ & $33.81 \pm 19.5$ & $9.0 \pm 3.3$ & $19 \pm 6.2$ & $14.4 \pm 2.8$ & $8.5 \pm 1.7$ \\
\hline $2 \min$ & 11 & $2.18 \pm 1.8^{*}$ & $5.5 \pm 1.3 *$ & $29.01 \pm 8.7$ & $8.27 \pm 1.7$ & $39.81 \pm 10.5$ & $7.27 \pm 2.6$ & $15.55 \pm 5.5$ & $17.27 \pm 3.5$ & $7.5 \pm 1.6$ \\
\hline $3 \min$ & 12 & $2.36 \pm 1.5 *$ & $5.0 \pm 1.5^{*}$ & $41.41 \pm 11.7^{*}$ & $4.8 \pm 1.3^{*}$ & $26.60 \pm 8.1$ & $4.89 \pm 1.8^{*}$ & $18.36 \pm 6.2$ & $17.0 \pm 4.6$ & $5.8 \pm 2.3^{*}$ \\
\hline $4 \mathrm{~min}$ & 15 & $5.09 \pm 2.7^{*}$ & $4.07 \pm 1.6^{*}$ & $33.41 \pm 7.5$ & $3.91 \pm 1.6^{*}$ & $30.52 \pm 19.4$ & $4.91 \pm 2.3^{*}$ & $11.73 \pm 6.2 *$ & $14.73 \pm 4.2$ & $6.0 \pm 2.0^{*}$ \\
\hline \multicolumn{11}{|l|}{ Striatum } \\
\hline $\mathrm{Co}$ & 10 & $1 \pm 0.9$ & $20.27 \pm 3.7$ & $34.28 \pm 11.2$ & $6.33 \pm 3.4$ & $192.94 \pm 74.5$ & $38.2 \pm 13.3$ & $22.41 \pm 7.1$ & $23.1 \pm 3.9$ & $47.0 \pm 52.1$ \\
\hline $2 \min$ & 11 & $1.45 \pm 1.3$ & $14.0 \pm 4.9 *$ & $23.55 \pm 8.00^{*}$ & $4.09 \pm 2.9$ & $134.55 \pm 35.9^{*}$ & $42.2 \pm 4.7$ & $22.65 \pm 12.8$ & $27.73 \pm 7.9$ & $97.0 \pm 20.3 *$ \\
\hline $3 \min$ & 12 & $1.45 \pm 1.6$ & $12.17 \pm 4.0 *$ & $43.35 \pm 11.9$ & $2.56 \pm 2.2 *$ & $118.34 \pm 35.5^{*}$ & $39.0 \pm 7.3$ & $41.5 \pm 28.6$ & $22.58 \pm 5.2$ & $102.83 \pm 17.2^{*}$ \\
\hline $4 \mathrm{~min}$ & 15 & $0.64 \pm 0.7$ & $16.87 \pm 5.2$ & $46.94 \pm 22.9$ & $2.22 \pm 1.5^{*}$ & $116.47 \pm 36.8^{*}$ & $29.2 \pm 13.3$ & $21.42 \pm 9.3$ & $16.3 \pm 6.3^{*}$ & $94.22 \pm 24.0^{*}$ \\
\hline \multicolumn{11}{|l|}{ Cortex } \\
\hline $\mathrm{Co}$ & 10 & $1.1 \pm 1.1$ & $38.0 \pm 6.2$ & $53.25 \pm 13.9$ & $1.56 \pm 1.5$ & $19.6 \pm 10.8$ & $56.1 \pm 12.9$ & $9.12 \pm 2.4$ & $30.78 \pm 8.5$ & $48.33 \pm 19.2$ \\
\hline $2 \min$ & 11 & $2.91 \pm 1.9^{*}$ & $38.25 \pm 7.6$ & $64.44 \pm 27.8$ & $3.27 \pm 3.77$ & $14.9 \pm 10.5$ & $64.0 \pm 11.0$ & $17.29 \pm 8.9^{*}$ & $36.91 \pm 8.9$ & $69.5 \pm 9.7^{*}$ \\
\hline $3 \mathrm{~min}$ & 12 & $3 \pm 1.9^{*}$ & $33.75 \pm 7.7$ & $74.62 \pm 16.2 *$ & $1.2 \pm 0.8$ & $11.64 \pm 10.2$ & $60.3 \pm 13.2$ & $23.35 \pm 6.5^{*}$ & $27.42 \pm 5.8$ & $65.5 \pm 12.2^{*}$ \\
\hline $4 \mathrm{~min}$ & 15 & $1.36 \pm 1.5$ & $34.93 \pm 5.5$ & $64.38 \pm 28.0$ & $1.31 .6 \pm 2.2$ & $12.3 \pm 8.2$ & $57.9 \pm 13.1$ & $21.33 \pm 7.5^{*}$ & $30.1 \pm 6.2$ & $69.00 \pm 7.2 *$ \\
\hline
\end{tabular}

$* p<0.05$.
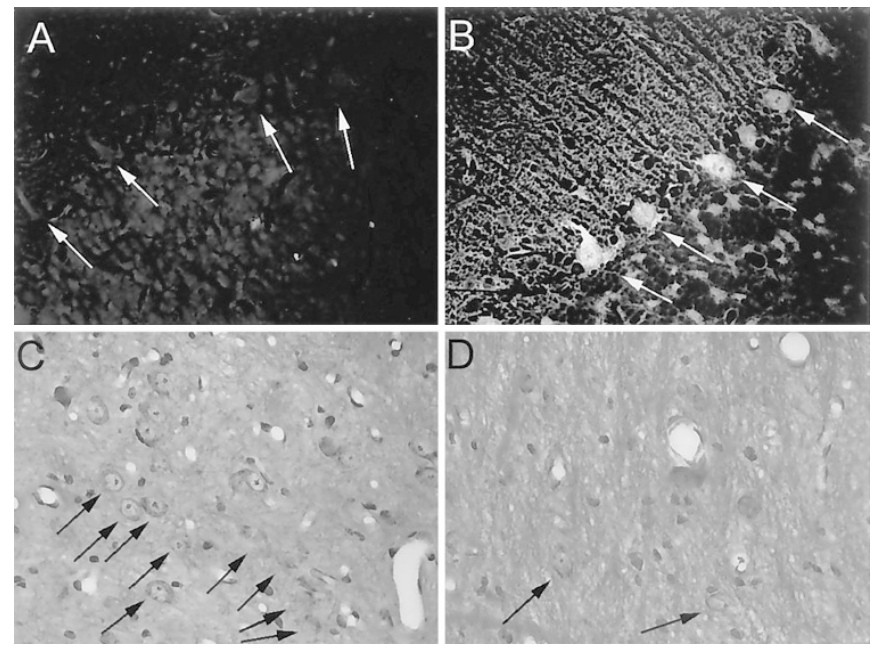

Figure 1. Cell degeneration and cell loss. Examples of stained paraffin sections of guinea pig brain used for morphometry, FJ staining in cerebellum $(A, B)$, and Nissl staining in hypothalamus $(C, D)$. Left images show control sections $(A, C)$, right images show cell degeneration and cell loss in animals after 4 min of perinatal asphyxia $(B, D)$. White arrows indicate Purkinje cells in FJ-stained sections. In PA brain, fibers, some Purkinje cells, and neurons in the granular layer showed a specific signal. In $C$ and $D$ Nissl staining revealed neurons in the area. The arrows in the lower part of the figure indicate neurons that were included for determination of cell density. Magnification, $\times 200$.

in the striatum after 3 min of PA. Results are shown in Table 2 .

Neurotransmitters. vMAT was decreased in the cerebellum and the striatum of all asphyctic groups (Table 2, and a representative staining pattern is shown in Fig. 2), and DBH

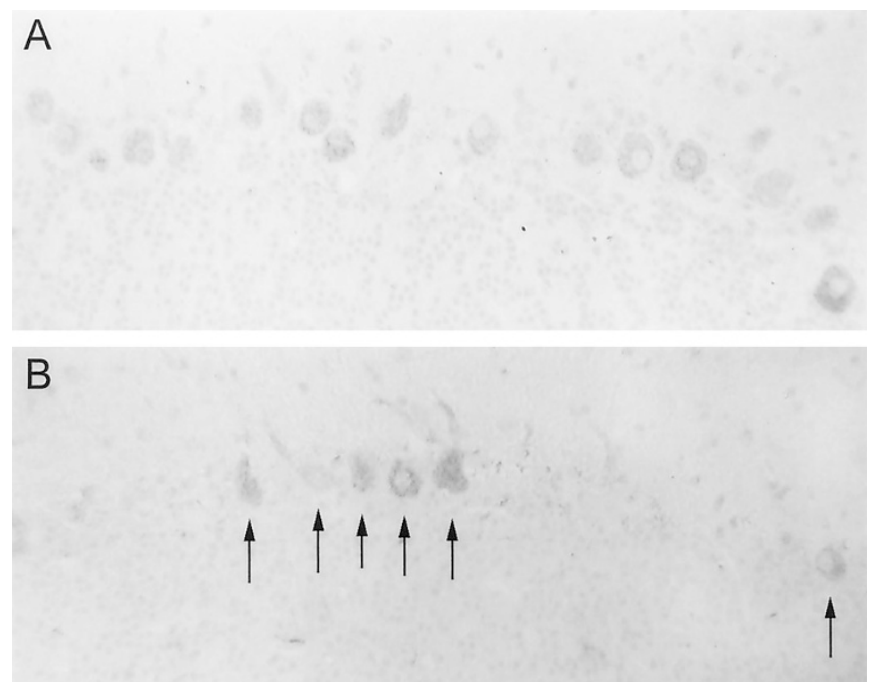

Figure 2. Immunostaining of vMAT in cerebellum as an example of perikarya evaluation. Paraffin sections of cerebellum stained for vMAT in control sections $(A)$ and after $4 \mathrm{~min}$ of PA $(B)$. In the lower image area, the arrows point out vMAT-expressing Purkinje cells that were included in the morphometric analysis. Note that in animals exposed to PA the number of vMAT-immunoreactive cells was reduced. Magnification, $\times 200$.

showed a decrease in the cerebellum and the hippocampus CA1 in the 4-min asphyctic animals, and in the hypothalamus (nucleus ventromedialis) at 3 and 4 min (Table 2). TH was decreased in all asphyctic groups in the cerebellum and the in hypothalamus at 4 min of PA, whereas it was significantly increased in the striatum in the 3-min asphyctic group and in 


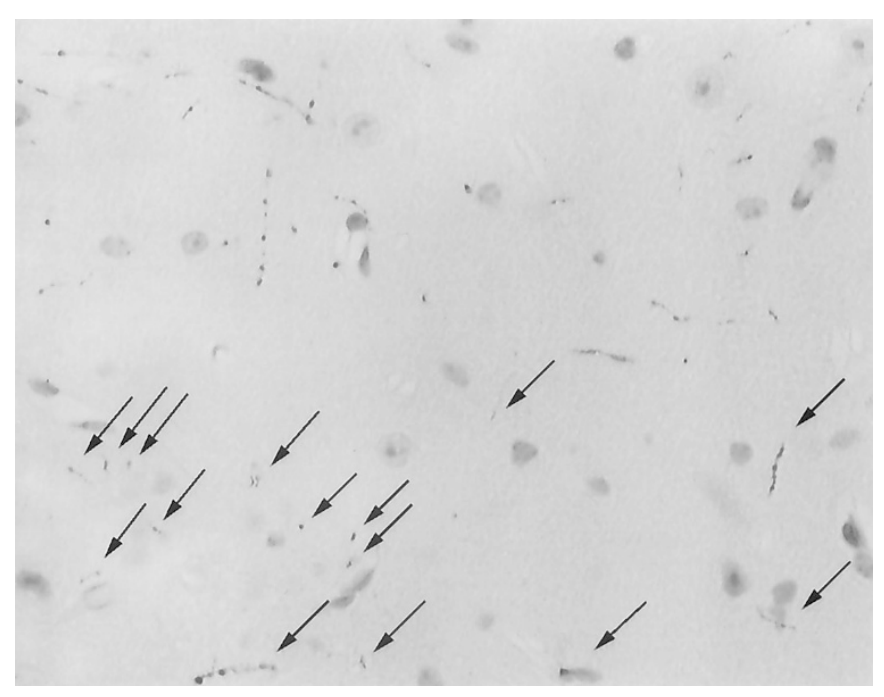

Figure 3. Immunostaining of $\mathrm{TH}$ in cortex as an example of nerve fiber evaluation. Cerebral cortex showing the molecular layer after TH immunostaining. The lower part of the image provides an example of how immunoreactive fibers were counted for morphometric analysis; each arrow points out a fiber included for fiber density evaluation as given in Table 2. Magnification, $\times 400$.

the parietal cortex of all asphyctic guinea pigs (Table 2, and a representative staining pattern is demonstrated in Fig. 3). EAAC1 presented with decreased immunoreactivity in the cerebellum, hippocampal area CA1, and the striatum after 4 min of PA (Table 2). vAChT was significantly increased in all asphyctic groups in the hippocampus, the striatum, and the parietal cortex and showed a decrease in the 3- and 4-min asphyctic groups in the cerebellum and the hypothalamus (Table 2).

\section{DISCUSSION}

Neurodegeneration with neuronal death, glial proliferation, and neurotransmitter changes has been shown to occur in various animal models of PA $(8-10,17,35-37)$. We now provide the first evidence for neurodegeneration in an animal model of PA 3 mo after delivery. FJ staining is a method correlating with silver staining protocols and reflecting neurodegeneration, including atrophy of dendritic spines and deficient arborization (7). FJ staining recognizes neurodegeneration in several pathologic processes including methamphetamine exposure or organotypic brain slice cultures treated with trimethyltin compounds, kainic acid, domoic acid, 3-nitropropionic acid, and 1-methyl-4-phenyl-1,2,3,6-tetrahydropyridine (7, 38, $39)$. By applying this method we clearly revealed neurodegenerative changes in several brain regions of asphyctic guinea pigs. We found degenerating FJ-positive neurons in the cerebellum, CA1, and the hypothalamus, although Nissl staining revealed neuronal loss in the hypothalamus and the cerebellum but not in CA1. Increased GFAP staining in the cerebellum and in the hippocampus paralleled the findings of FJ-positive degenerating neurons.

Neuroblastoma cells that morphologically present with variable degrees of differentiation, including neuritic expanding processes and sinus formation with distant cells, showed NSP-
C-dependent differentiation. Hens and coworkers (40) recently showed that highly differentiated neuronal cell lines expressed NSP-C to a higher extent than cell lines showing less neuronal differentiation. Moreover, induction of neuronal differentiation in undifferentiated pheochromocytoma PC12 cells induced a strong increase in NSP-C expression. Significant reduction of NSP-C was a prominent finding in the hypothalamus and the striatum, and we present the novel finding that differentiation is impaired in PA. We believe from this observation that different brain regions show different susceptibilities to the development of neurodegeneration, neuronal loss, and differentiation.

Evaluation of the neurotransmitter systems confirmed this statement. The monoaminergic system as represented by vMAT, DBH, and $\mathrm{TH}$ was not affected in all regions studied but at least one of the three monoaminergic markers was deranged in at least one area, suggesting that all three markers would have to be applied in all studies on this neurotransmitter system. Also deterioration of the monoaminergic system was not regularly accompanied by changes in glutamatergic or cholinergic innervation.

When comparing our guinea pig data with those of the (immature) rat animal model of PA at the identical time point, we found differences in terms of affected regions and involvement of individual neurotransmitters (23), indicating speciesspecific differences. When comparing our results in the guinea pig with the human system, we found neuronal loss, gliosis, and monoaminergic and cholinergic changes in areas affected also in the asphyxiated infant (31-33), although not all areas with lesions in the human system were studied and results are not fully congruent. Furthermore, no FJ-staining data, now an accepted standard assay for neurodegeneration, are available in the asphyxiated infant and no differentiation markers were studied in humans with PA. Also information about the glutamatergic system in human PA is virtually missing. We therefore propose that the model of PA in the guinea pig resembles human PA in several aspects and thus warrant studies on NSP-C and FJ in the human brain with PA.

Differences in pathologic mechanisms and neuropathology among mammalian species may be explained by individual susceptibility of species to hypoxia-ischemia, which in turn is known to depend on metabolism, basal metabolic rate, oxygen consumption, and different mechanisms to cope with hypoxiaischemia including hypoxia-inducible factors. We are aware of the inherent problem of comparing rats, humans, and guinea pigs in terms of metabolism, susceptibility to hypoxia, and so forth, but we must point out the importance of the state of maturity or immaturity - although our findings clearly discriminate between consequences of PA in the rat and those in the guinea pig. Rats in our model of PA survive 20 min of hypoxia, whereas the guinea pig only tolerates 4 min of the hypoxic state $(25,26)$. The guinea pig is a developmentally mature animal, able to follow the pack shortly after delivery, whereas rats and humans are less mature or even poorly developed at birth. It has already been proposed that the mature brain is more susceptible to hypoxic-ischemic brain damage (41), and the higher susceptibility of the mature animal may not only be reflected by metabolic function but also by the different capabilities of the immature mechanism to performing repair pro- 
cesses and, moreover, to possess an increased potential for proliferation of neurons.

\section{CONCLUSIONS}

We show that the guinea pig develops neurodegeneration after PA as revealed by FJ staining, and presents with neuronal loss, glial proliferation, and dedifferentiation. The nature of the neuronal loss, including impaired monoaminergic, glutamatergic, and cholinergic innervation, is presented and discussed as being different from that of the rat with PA. Our work is relevant because it reflects a simple animal model of PA resembling intrauterine perinatal asphyxia without ligation of brain vessels, use of chemical hypoxia, mechanical ventilation and so forth, leading to neurodegeneration as seen in the human system evaluated by classical neuropathologic criteria, although not all affected brain regions were comparable between guinea pig and human. Although neurodegeneration is unequivocally shown in the guinea pig model, direct implications for the human neonate cannot be absolutely extrapolated. Studies on additional regions as well as different time points are necessary as are studies on neurodegeneration using $\mathrm{FJ}$, NSP-C, and other stains in the human brain with PA to enable further comparison between the guinea pig model and the asphyxiated child.

Acknowledgment. We thank Prof. Dr. Gert Lubec, FRSC (UK) for scientific advice.

\section{REFERENCES}

1. Hill A 1991 Current concepts of the hypoxic-ischemic cerebral injury in the term newborn. Pediatr Neurol 7:317-325

2. Volpe JJ 2001 Hypoxic-ischemic encephalopathy: biochemical and physiological aspects. In: Volpe JJ (ed) Neurology of the Newborn. WB Saunders, Philadelphia, pp 217-276

3. Younkin DP 1992 Hypoxic-ischemic brain injury of the newborn-statement of the problem and overview. Brain Pathol 2:209-210

4. Bottiger BW, Schmitz B, Wiessner C, Vogel P, Hossman KA 1998 Neuronal stress response and neuronal cell damage after cardiocirculatory arrest in rats. J Cereb Blood Flow Metab 18:1077-1087

5. Laptook AR, Corbett R, Sterett R, Burns DK, Tollefsbol G, Garcia D 1994 Modest hypothermia provides partial neuroprotection for ischemic neonatal brain. Pediatr Res 35:436-442

6. Thompson CB 1995 Apoptosis in the pathogenesis and treatment of disease. Science 267:1456-1462

7. Schmued LC, Bowyer JF 1997 Methamphetamine exposure can produce neuronal degeneration in the mouse hippocampal remnants. Brain Res 795:135-140

8. El Khodor BF, Boksa P 1997 Long term reciprocal changes in dopamine levels in prefrontal cortex versus nucleus accumbens in rats born by Caesarean section compared to vaginal birth. Exp Neurol 145:118-129

9. Blennow M, Zeman J, Dahlin I, Lagercrantz H 1995 Monoamine neurotransmitters and metabolites in the cerebrospinal fluid following perinatal asphyxia. Biol Neonate 67:407-413

10. Chen Y, Engidawork E, Loidl F, Dell'Anna E, Goiny M, Lubec G, Andersson K, Herrera-Marschitz M 1997 Short- and long term effects of perinatal asphyxia on monoamine, amino acid and glycolysis product levels in the basal ganglia of the rat. Brain Res Dev Brain Res 104:19-30

11. Chen Y, Herrera-Marschitz M, Bjelke B, Blum M, Gross J, Andersson K 1997 Perinatal asphyxia induced changes in rat brain tyrosine hydroxylase immunoreactive cell body number: effects of nicotine treatment. Neurosci Lett 221:77-80

12. Ungethum U, Chen Y, Gross J, Bjelke B, Bolme P, Eneroth P, Heldt J, Loidl CF, Herrera-Marschitz M 1996 Effects of perinatal asphyxia on the mesostriatal/ mesolimbic dopamine system of neonatal and 4-week-old male rats. Exp Brain Res 112:403-410
13. Chen Y, Ogren SO, Bjelke B, Bolme P, Eneroth P, Gross J, Loidl F, HerreraMarschitz M 1995 Nicotine treatment counteracts perinatal asphyxia induced changes in the mesostriatal/mesolimbic dopamine system and in motor behavior in the four week old male rat. Neuroscience 68:531-538

14. Bjelke B, Andersson K, Ogren SO, Bolme P 1991 Asphyctic lesions: proliferation of tyrosine hydroxylase immunoreactive nerve cell bodies in the rat substantia nigra and functional changes in dopamine neurotransmission. Brain Res 543:1-9

15. Burke RE, Karanas AL 1990 Quantitative morphological analysis of striatal cholinergic neurons in perinatal asphyxia. Ann Neurol 27:81-88

16. Otoya RE, Seltzer AM, Donoso AO 1997 Acute and long-lasting effects of neonatal hypoxia on (+)-3-[125I]MK-801 binding to NMDA brain receptors. Exp Neurol 148:92-99

17. Fritz KI, Groenendaal F, McGown JE, Mishra OP, Delivoria-Papadopoulos M 1996 Effect of cerebral hypoxia on NMDA receptor binding characteristics after treatment with 3-(2-carboxypiperazin-4-yl) propyl-1-phosphonic acid (CPP) in newborn piglets. Brain Res 729:66-74

18. Martin LJ, Brambrink AM, Lehmann C, Portera-Cailliau C, Koehler R, Brothstein J, Traystman RJ 1997 Hypoxia-ischemia causes abnormalities in glutamate transporters and death of astroglia and neurons in newborn striatum. Ann Neurol 42:335-348

19. Cai Z, Sogrest C, Hersey K, Rodes PG 1995 Intrauterine hypoxia-ischemia increases $\mathrm{N}$-methyl-D-aspartate-induced cGMP formation and glutamate accumulation in cultured rat cellular granule cells. Pediatr Res 38:107-112

20. Gilland E, Puka-Sundvall M, Andine P, Bona E, Hagberg H 1994 Hypoxic-ischemic injury in the neonatal rat brain: effect of pre- and posttreatment with the glutamate release inhibitor BW1003C87. Brain Res Dev Brain Res 83:79-84

21. Sher PK 1991 2-AVP and DGAMS are superior to MK-801 in preventing hypoxiainduced injury to developing neurons in vitro. Pediatr Neurol 7:429-435

22. Silverstein FS, Naik B, Simpson J 1991 Hypoxia-ischemia stimulates hippocampal glutamate efflux in perinatal brain: an in vivo microdialysis study. Pediatr Res 30:587-590

23. Kohlhauser C, Kaehler S, Mosgoeller W, Singewald N, Prast H, Hoeger H, Lubec B 1999 Histological changes and neurotransmitter levels three months following perinatal asphyxia in the rat. Life Sci 64:2109-2124

24. Kohlhauser C, Mosgoeller W, Hoeger H, Lubec G, Lubec B 1999 Cholinergic monoaminergic and glutamatergic changes following perinatal asphyxia in the rat Cell Mol Life Sci 55:1491-1501

25. Lubec B, Dell'Anna E, Fang-Kircher S, Marx M, Herrera-Marschitz M, Lubec G 1997 Decreased brain protein kinase C, PKA and cyclin-dependent kinase precedes neuronal death in perinatal asphyxia in the rat. J lnvestig Med 45:284-294

26. Lubec B, Marx M, Herrera-Marschitz M, Labudova O, Hoeger H, Gille L, Nohl H, Mosgoeller W, Lubec G 1997 Decrease of heart protein kinase C and cyclindependent kinase precedes death in perinatal asphyxia of the rat. FASEB J 11:482491

27. Lubec B, Chiappe-Gutierrez M, Hoeger H, Kitzmueller E, Lubec G 2000 Glucose transporters, hexokinase, and phosphofructokinase in brain of rats with perinatal asphyxia. Pediatr Res 47:84-88

28. Marx M, Muhl A, Hoeger H, Rolinski B, Frisch H, Herkner K, Lubec G, Lubec B 2001 Brain fatty acids in perinatal asphyxia. Life Sci 68:2657-2664

29. Dell'Anna ME, Chen Y, Engidawork E, Andersson K, Lubec G, Luthman J, HerreraMarschitz M 1997 Delayed neuronal death following perinatal asphyxia in the rat Exp Brain Res 5:301-309

30. Burck HC 1981 Histologische Technik, 4th Ed. Georg Thieme Verlag, Stuttgart

31. Volpe JJ 2001 Hypoxic-ischemic encephalopathy: neuropathology and pathogenesis. In: Volpe JJ (ed) Neurology of the Newborn. WB Saunders, Philadelphia, pp 296-330

32. Friede RL 1989 Developmental Neuropathology, 2nd Ed. Springer Verlag, New York, pp 82-97

33. Barkovich A $1992 \mathrm{MR}$ and CT evaluation of profound neonatal and infantile asphyxia. AJNR Am J Neuroradiol 13:959-972

34. Roessner W 1965 Stereotaktischer Hirnatlas vom Meerschweinchen. Pallas Verlag, Lochham bei Muenchen, pp 6-26

35. Northington FJ, Ferriero DM, Graham EM, Traystman RJ, Martin LJ 2001 Early neurodegeneration after hypoxia-ischemia in neonatal rat is necrosis while delayed neuronal death is apoptosis. Neurobiol Dis 8:207-219

36. Northington FJ, Ferriero DM, Martin LJ 2001 Neurodegeneration in the thalamus following neonatal hypoxia-ischemia is programmed cell death. Dev Neurosci 23:186-191

37. Martin LJ 2001 Neuronal cell death in nervous system development, disease, and injury. Int J Mol Med 7:455-478

38. Noraberg J, Kristense BW, Zimmer J 1999 Markers for neuronal degeneration in organotypic slice cultures. Brain Res Brain Res Protoc 3:278-290

39. Schmued LO, Albertson C, Slikker W 1997 Fluoro-Jade: a novel fluorochrome for the sensitive and reliable histochemical localization of neuronal degeneration. Brain Res $751: 37-46$

40. Hens J, Nuydens R, Geerts H, Senden NH, van de Ven WJ, Roebrok AJ, van de Velde HJ, Ramaekers FC, Broers JL 1998 Neuronal differentiation is accompanied by NSP-C expression. Cell Tissue Res 292:229-237

41. Yager JY, Thornhill JA 1997 The effect of age on susceptibility to hypoxic-ischemic brain damage. Neurosci Biobehav Rev 21:167-174 